\title{
Biodiversidad de
}

\section{macroinvertebrados acuáticos} y la calidad del agua en la subcuenca de los ríos Bul Bul y Paiwas, Matiguás, Nicaragua

Aura Y. Cárdenas ${ }^{1}$, Bismark Reyes ${ }^{2}$, Marlon López ${ }^{3}$, Alfredo Woo ${ }^{4}$, Elías Ramírez ${ }^{5}$ y Muhammad Ibrahim ${ }^{6}$

1 Mon. Agua y SIG. Proyecto GEF-Silvopastoril, Instituto de Investigación y Desarrollo Nitlapan, Universidad Centroamericana. E-mail: auracard@ns.uca.edu.ni, aycardenas@gmail.com

2 Asistente local para monitoreo de calidad de agua en microcuencas ganaderas.

3 Coordinador proyecto BNPP, CATIE Turrialba, Costa Rica.

4 Asistente local para monitoreo de usos de suelo

5 Coordinador Nacional Proyecto GEF-Silvopastoril, Instituto de Investigación y Desarrollo Nitlapan, Universidad Centroamericana. E-Mail: nitlactaf@ns.uca.edu.ni,

6 Coordinador del grupo Gamma, CATIE.E-mail: mibrahim@catie.ac.cr

Recibido: junio de 2007 / Aceptado: julio de 2007

EN ESTE ESTUDIO SE DETERMINÓ LA DIVERSIDAD BIOLÓGICA de macroinvertebrados acuáticos asociada a los usos de suelo más comunes en la zona ganadera de Matiguás, específicamente: pastura mejorada con alta densidad de árboles (PMADA), pastura mejorada con baja densidad de árboles (PMBDA), bosque ripario (BR), banco forrajero $(\mathrm{BF})$ y pastura degradada (PD). Se midió el efecto de los usos de suelo en la calidad del agua. Para esto se monitorearon 6 quebradas en la microcuenca del río Paiwas y 3 en el río Bul Bul. El número total de taxas encontradas fue de 15 órdenes, predominando los órdenes Efemeróptero (39\%) y Díptera (18\%); 51 familias y 82 géneros, de los cuales el 91\% pertenecen a la clase insecta. En lo que se refiere a calidad de agua se determinaron 3 grupos; uno que reúne organismos indicadores de buena calidad de agua, otro para organismos indicadores de mediana calidad y por último los organismos indicadores de mala calidad. El componente de protección en las quebradas fue clave para la diversidad biológica, evidenciándose diferencias significativas $(\mathrm{p}<0,05)$ entre las quebradas que presentan protección y las desprotegidas.

Palabras clave: diversidad biológica-investigaciones / ganadería-Matiguás, Matagalpa (Nicaragua) / calidad del agua 


\section{Introducción}

Existe un creciente interés por conocer la manera de proteger los sistemas fluviales y estudiar sus cambios en el tiempo, lo que ha estimulado el desarrollo de criterios biológicos que permitan estimar el efecto de las intervenciones humanas en éstos (Figueroa et al., 2003). La mayoría de estos estudios se realiza con la finalidad de tomar medidas en la conservación y protección de las fuentes de agua, y mejorar la cantidad y calidad de la misma.

Cuando se evalúa un sistema acuático para diferentes fines es necesario realizar un análisis integral donde se incluyan análisis físico-químicos, bacteriológicos y biológicos, así como de los diferentes usos de suelo que forman parte de las microcuencas. La información biológica no reemplaza los registros físicos y químicos para definir la calidad del agua, especialmente la asociada al crecimiento poblacional; esta información es característica de un medio ambiente que cuantifica el nivel de exposición y el grado de respuesta ecológica ante dicha situación (De la Lanza et al., 2000).

En varios estudios se evidencia que la deforestación y la ganadería extensiva afectan a la biota presente en los sistemas acuáticos debido al uso de agroquímicos, el aumento de sedimentos, la pérdida de la vegetación ribereña, el aumento de la temperatura del agua, la menor regulación de caudales y el daño que el ganado provoca al cauce y a los taludes (Chará, 2003) al tener un acceso directo y constante a las fuentes de agua (Auquilla, 2005).

Las fincas ganaderas con sistemas silvopastoriles como árboles dispersos en las pasturas, bancos forrajeros y cercas vivas, contribuyen a disminuir el impacto de la contaminación (Auquilla, 2005), aumentan la capacidad de retención de agua en las praderas, ayudan a la infiltración, y protegen el suelo, los manantiales y las quebradas (Chará, 2003). Es por eso que la planificación de fincas ganaderas en función de la protección de los recursos existentes puede contribuir a regular y conservar los recursos hídricos mediante la arborización, la adopción de sistemas silvopastoriles (SSP) y de prácticas de conservación de suelos.

En este estudio se determinó la biodiversidad de macroinvertebrados acuáticos y la calidad del agua asociada a los usos de suelo más comunes dentro de la zona ganadera de Matiguás, Matagalpa.

\section{Metodología}

\subsection{Descripción del área de estudio}

El estudio se realizó en Matiguás en la subcuenca de los ríos Bul Bul y Paiwas, vertiente del Atlántico, con una topografía que oscila entre los 200 y 400 metros sobre el nivel del mar. Se localiza entre las coordenadas $85^{\circ}$ y $27^{\circ}$ de latitud norte, $12^{\circ}$ y $50^{\circ}$ de longitud oeste (Ilustración 1). Posee un régimen de lluvias que varía entre 1,200 y 1,800 mm. anuales, con una distribución de mayo a diciembre (Guerrero \& Soriano, 1992). Los usos de suelo que predominan son los pastizales y tacotales, y la principal actividad económica es la ganadería de leche y carne (Ruíz, 2002). 


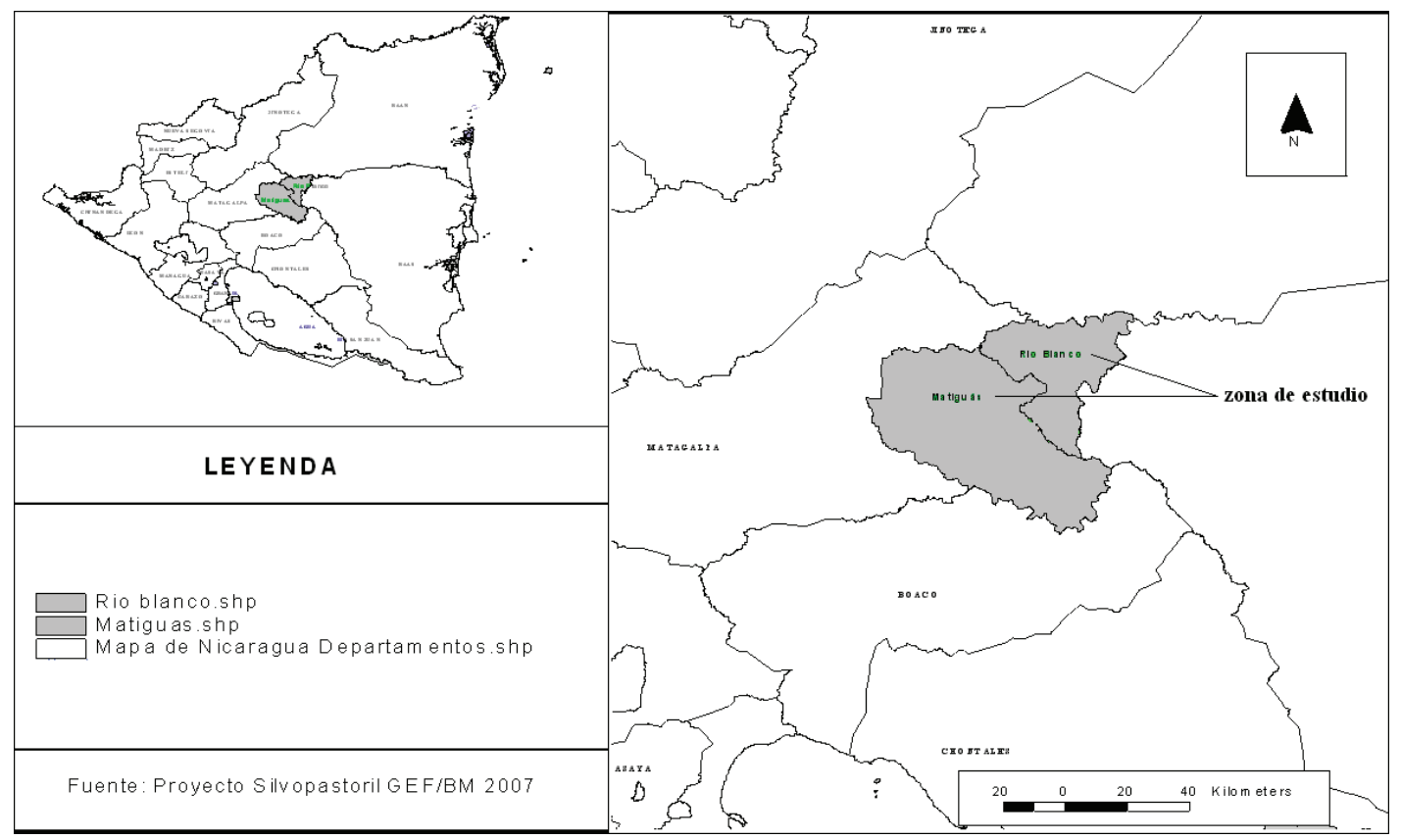

Ilustración 1. Mapa de ubicación de la zona de estudio.

\subsection{Selección de las quebradas}

Los usos de suelo que se estudiaron fueron los más predominantes en el área de estudio, específicamente: pasturas mejoradas con alta densidad de árboles (PMADA), bancos forrajeros (BF), pasturas mejoradas con baja densidad de árboles (PMBDA) y pasturas degradadas (PD). Esta información fue obtenida del monitoreo de usos de suelo realizado por el Proyecto Regional Enfoques silvopastoriles integrados para el manejo de ecosistemas, en el cual se contempló la elaboración de los perímetros de las fincas, el registro de usos de suelo, la evolución histórica de la finca y el monitoreo de los cambios en el uso del suelo durante 5 años.

Se seleccionaron 9 quebradas (5 protegidas por bosque ripario y 4 sin protección) ubicadas en dos subcuencas: 6 quebradas en la subcuenca del río Paiwas y 3 quebradas en la subcuenca del río Bul Bul, (Cuadro 1). 
Cuadro 1. Descripción general de las fuentes de agua que se monitorearon en la cuenca de los ríos Paiwas y Bul Bul, Matiguás, Nicaragua (2005- 2006).

\begin{tabular}{|c|c|c|c|}
\hline $\begin{array}{l}\text { Sub- } \\
\text { cuenca }\end{array}$ & Quebrada & Descripción General & $\begin{array}{l}\text { Longitud de las } \\
\text { quebradas (m) }\end{array}$ \\
\hline \multirow[t]{6}{*}{ Paiwas } & 1 & $\begin{array}{l}\text { Zona sin protección, dentro de banco } \\
\text { forrajero }\end{array}$ & 310 \\
\hline & 2 & $\begin{array}{l}\text { Protegido por bosque ripario, pastura } \\
\text { mejorada con alta densidad de árboles }\end{array}$ & 605 \\
\hline & 3 & $\begin{array}{l}\text { Protegido por bosque ripario, pastura } \\
\text { mejorada con alta densidad de árboles }\end{array}$ & 613 \\
\hline & 4 & $\begin{array}{l}\text { Zona sin protección, en pasturas, zona de } \\
\text { pastoreo, pasturas mejoradas con baja } \\
\text { densidad de árboles }\end{array}$ & 196 \\
\hline & 5 & $\begin{array}{l}\text { Protegido por bosque ripario, pastura } \\
\text { degradada }\end{array}$ & 1005 \\
\hline & 6 & $\begin{array}{l}\text { Desprotegida en la naciente, pasturas } \\
\text { degradadas }\end{array}$ & 465 \\
\hline \multirow[t]{3}{*}{ Bul Bul * } & 7 & $\begin{array}{l}\text { Protegido por bosque ripario, pastura } \\
\text { mejorada con alta densidad de árboles }\end{array}$ & 498 \\
\hline & 8 & $\begin{array}{l}\text { Protegido por bosque ripario, pastura } \\
\text { mejorada con alta densidad de árboles }\end{array}$ & 1059 \\
\hline & 9 & $\begin{array}{l}\text { Cultivos, sin protección, piscina (tilapias), } \\
\text { pastos mejorados, residuos de vivienda }\end{array}$ & 305 \\
\hline
\end{tabular}

*Estas quebradas están ubicadas cerca de la zona de amortiguamiento de un área protegida cuya composición es bosque nativo.

Las estaciones para muestrear macroinvertebrados bentónicos se seleccionaron considerando las siguientes variables:

- Quebradas con flujo permanente (se evitaron quebradas intermitentes)

- Fincas con diferentes usos de suelo: pasturas mejoradas con alta densidad de árboles, pasturas mejoradas sin árboles, banco forrajero de gramíneas y pasturas degradadas.

En cada quebrada se seleccionaron 2 puntos para recolectar macroinvertebrados acuáticos, y con el propósito de evaluar de manera integral los cuerpos de agua, se tomaron datos in situ de oxígeno disuelto $(\mathrm{mg} / \mathrm{l})$, potencial Hidrógeno $(\mathrm{pH})$, temperatura $\left({ }^{\circ} \mathrm{C}\right)$, conductividad eléctrica (ms/cm), y velocidad del caudal, utilizando un equipo portátil HACH, así:

- El primer punto a $50 \mathrm{~m}$ hacia abajo de la naciente

- El segundo punto a $50 \mathrm{~m}$ hacia abajo en la zona de los potreros

Los muestreos se realizaron en los meses de agosto, septiembre y noviembre (época lluviosa); febrero y mayo (época seca). La zona de muestreo incluyó los diferentes microhábitats de las quebradas (pozos, corrientes rápidas y lentas). Se escogió un área equivalente al ancho 
húmedo promedio de la quebrada por unos 3 metros de largo para recoger información de los microhábitats.

\subsection{Análisis biológico de la calidad del agua}

En el muestreo de organismos bentónicos para la época seca se colectó un total de 24 muestras en 6 estaciones de monitoreo y 2 puntos de muestreo. En la época lluviosa se colectó un total de 36 muestras en 9 estaciones de monitoreo y 2 puntos de muestreo.

Se utilizó la metodología aplicada por García (2003), quien recomienda que los sitios de muestreo sean representativos de los diferentes usos del suelo y actividades humanas. Para recolectar las muestras de macroinvertebrados se realizaron arrastres en un tiempo de 5 minutos por los micro-hábitat identificados en el sitio, como pozos, corrientes rápidas y lentas, removiendo el fondo del río y colectando el material removido en los $50 \mathrm{~m}$ seleccionados utilizando una red de mano tipo "D" con malla de $500 \mu \mathrm{m}$. y apertura de 20 a $25 \mathrm{~cm}$. Además se tomaron datos de la morfología del cauce (sustratos y componentes de la quebrada como hojas, piedras, ramas y presencia de sombra) para caracterizar los distintos microhábitats colonizados por los macroinvertebrados.

La separación de los organismos bentónicos se realizó en el campo colocando la muestra en una bandeja blanca, y por medio de pinzas, retirando todos los macroinvertebrados presentes para luego colarlos en frascos plásticos con alcohol al 70\% debidamente rotulado. La identificación se hizo en la zona de estudio utilizando las claves de Roldán (1988) y preservando las muestras en alcohol al 70\% en viales.

\subsection{Análisis estadístico}

De manera general se hizo uso de estadística descriptiva, análisis multivariado por conglomerado, para agrupar los usos de suelo en base a los niveles de calidad de agua: pastura mejorada con alta densidad de árboles (PMADA), pastura mejorada con baja densidad de árboles (PMBDA), bosque ripario (BR), pastura degradada (PD), banco forrajero (BF).

Para determinar la diversidad biológica se utilizó el índice de diversidad Shannon Weaver (Shannon \& Weaver, 1949), pues en primer lugar, expresa la diversidad como función del número de relaciones que pueden construirse entre determinadas clases de elementos; y en segundo lugar, la expresión factorial que usa es muy apropiada para demostrar los cambios que experimenta la diversidad como consecuencia de alteraciones en el ecosistema.

$$
\begin{aligned}
& \text { Donde: } \mathrm{H}^{\prime}=\Sigma(\text { ni/N) } \ln (\text { ni/N) } \\
& \mathrm{i}=1
\end{aligned}
$$


Para agrupar los macroinvertebrados en base a la calidad del agua, tomando en cuenta la protección de las quebradas y la identificación de los usos de suelo que contribuyen a mejorar la calidad del agua, se utilizó el índice biológico de calidad BMWP (Biological Monitoring Working Party), un método rápido de valoración de la calidad biológica de un cuerpo de agua que utiliza los macroinvertebrados como indicadores (Roldán, 1988).

Se aplicó la Sumatoria de Ephemeroptera, Plecoptera y Trichoptera ( $\Sigma \mathrm{EPT})$, que se utiliza como un índice de riqueza, porque proporciona información rápida del estado de conservación de las aguas, siendo éste el más indicado para aplicar en casos de diagnósticos rápidos y seguimiento cuando se pretende hacer restauración de las fuentes de agua (Chará, 2003).

\section{Resultados y discusión}

Se registró un total de 4,093 individuos de macroinvertebrados agrupados en 15 órdenes, 51 familias y 82 géneros. El 91\% de los géneros pertenece a la clase Insecta y el 9\% restante a otros grupos como Moluscos, Decápoda, Tricládida Annélida, Hirudíneas. Los individuos del orden Efemeróptera predominaron con un (39\%), las familias de este orden se encuentran en aguas medianamente limpias y oxigenadas, aunque algunas especies resisten cierto grado de contaminación y son colonizadoras de otros hábitats. Otro grupo representativo fue Díptera (18\%), característico de zonas ganaderas y hábitats variados (Ilustración 2).

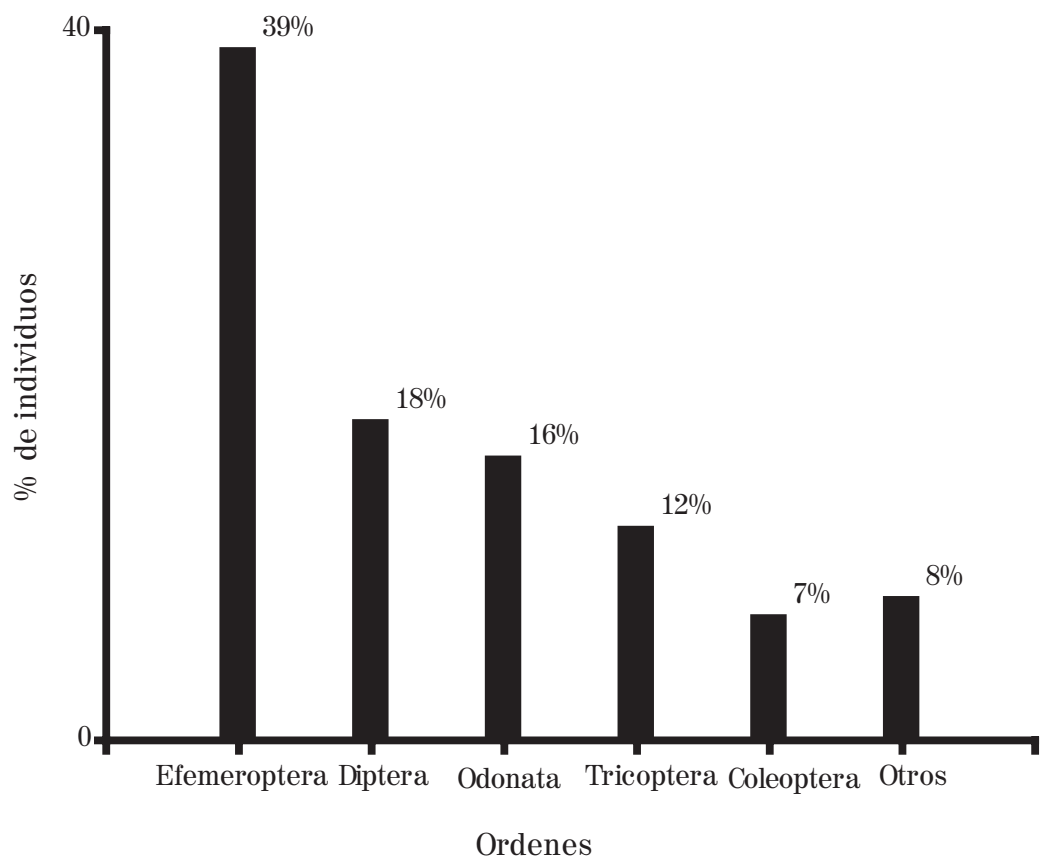

Ilustración 2. Porcentajes de órdenes de macroinvertebrados encontrados en las quebradas muestreadas en la subcuenca de los ríos Bul Bul y Paiwas, Matiguás, Nicaragua, (20052006) 
El Cuadro 2 refleja la agrupación de los macroinvertebrados acuáticos más sensibles a la perturbación y que tienden a disminuir en diversidad o en número cuando las condiciones no son favorables. Este grupo, $\Sigma$ EPT se encuentra bien representado en las quebradas protegidas con un $76 \%$ del total de individuos registrados, mostrando que el hábitat proporcionado por las quebradas protegidas es óptimo para albergar organismos indicadoras de buena calidad de agua.

Cuadro 2. Distribución de individuos macroinvertebrados acuáticos en las quebradas protegidas y no protegidas estudiadas en Matiguás, Nicaragua (2005-2006)

\begin{tabular}{|l|l|l|l|}
\hline Variable & $\begin{array}{l}\text { Quebradas } \\
\text { no protegidas }(\mathrm{n}=5)\end{array}$ & $\begin{array}{l}\text { Quebradas } \\
\text { protegidas }(\mathrm{n}=4)\end{array}$ & $\mathrm{P}$ \\
\hline No. de taxa total & 1971 & 2122 & $<005$ \\
\hline No. de taxa de Efemeroptera & 325 & 1105 & $<005$ \\
\hline No. de taxa de Plecoptera & 6 & 61 & $<005$ \\
\hline No. de taxa de Trichoptera & 64 & 447 & $<005$ \\
\hline No. de taxa de EPT" & 395 & 1613 & $<005$ \\
\hline Porcentaje de EPT" & $20 \%$ & $76 \%$ & $<005$ \\
\hline "EPT= Sumatoria de los órdenes Efemeróptera, Plecóptera y Trichóptera. \\
\hline
\end{tabular}

Con el análisis por conglomerado utilizando el índice BMWP (Ilustración 3), se ubicaron los usos de suelo estudiados de acuerdo a 3 categorías de calidad de agua: buena calidad, mediana calidad y mala calidad.

PMADA = pastura mejorada con alta densidad de árboles, $\mathrm{PM}=$ pastura mejorada con baja densidad de árboles, $\mathrm{BF}=$ banco forrajero, $\mathrm{BR}=$ bosque ripario $\mathrm{PD}=$ pastura degrada.

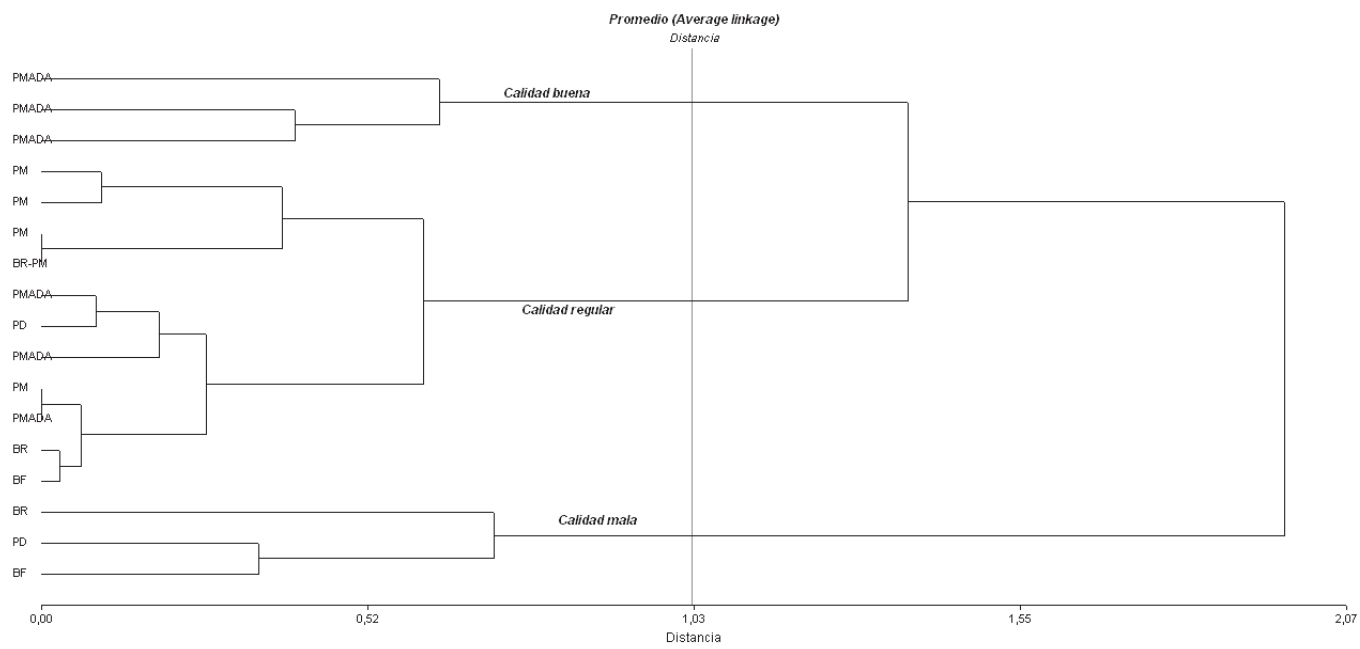

Ilustración 3. Resultados del análisis multivariado por conglomerado que muestra tres agrupaciones para el índice BMWP según la calidad de agua de cada uso de suelo estudiados en Matiguás, Nicaragua (2005 - 2006). 


\section{Grupo 1: Buena calidad}

Los organismos indicadores de buena calidad de agua se encuentran en ambientes poco perturbados, son principalmente Efemeroptera, Plecoptera y Trichoptera. En este estudio la mayoría de estos organismos se encontraron asociados a quebradas ubicadas en la microcuenca del río Bul Bul en pasturas mejoradas con alta densidad de árboles (PMADA) y una franja de bosque ripario como protección. Además, estos usos de suelo están ubicados cerca de la zona de amortiguamiento de bosques secundarios y primarios, reflejando el efecto positivo que tiene la combinación de pastos con vegetación arbustiva y arbórea en la salud del ambiente acuático, tanto en flora, fauna, como en calidad del agua, mostrando que esta asociación permite atrapar sedimentos y nutrientes antes de que lleguen a las corrientes.

\section{Grupo 2: Mediana calidad}

Los organismos indicadores de mediana calidad de agua o ambientes medianamente perturbados son principalmente Hemíptero, Odonata y Coleóptero. Se encontraron asociados a las quebradas de Paiwas y a los siguientes usos de suelo: pasturas mejoradas con baja densidad de árboles (PMBDA), pastura degradada (PD) con franjas de bosques riparios que protegen a las quebradas, pastura mejorada con alta densidad de árboles (PMADA), bosque ripario (BR) y banco forrajero (BF), con una cobertura de suelo de mas del $85 \%$.

Las quebradas asociadas a estos usos de suelo resultaron vulnerables a la contaminación por el desarrollo de actividades pecuarias en la zona, el acceso directo del ganado a las fuentes de agua, y en algunos casos, la deposición directa de materia fecal. Un buen manejo de la ganadería y control en el uso de herbicidas contribuirían a mejorar su calidad.

\section{Grupo 3: Mala calidad}

Los organismos indicadores de mala calidad o ambientes perturbados son principalmente Díptera y Mollusca, que se encontraron asociados a los bancos forrajeros (BF) y bosques riparios (BR) con mucha intervención del ganado y actividades agrícolas. Aunque los bancos forrajeros se encuentran también en el grupo de mediana calidad, al momento que se empieza con las labores de corte y acarreo hay una intervención directa y con ello la destrucción del hábitat, proporcionando las condiciones necesarias para el establecimiento de especies resistentes a la perturbación.

\subsection{Diversidad biológica}

Se encontró mayor diversidad de especies en aguas de buena calidad (Cuadro 3). Estos resultados son respaldados por varios estudios en los que se dice que las aguas de buena calidad son los que poseen mayor diversidad biológica (Auquilla, 2005; Chará, 2003).

En el cluster de mediana y mala calidad de agua se encontró una baja diversidad de macroinvertebrados acuáticos (Cuadro 3). Esto se debe principalmente a que no hay hábitats propicios para albergar grandes cantidades de individuos, ya que los parámetros 
fisicoquímicos encontrados, específicamente de Oxígeno disuelto (OD2), estaban por debajo de los niveles permisibles (6.5mg/L). La concentración de oxígeno es relevante en el control de la calidad de las aguas, siendo su presencia y concentración esencial para sustentar las formas superiores de vida. El comportamiento de estos dos grupos fue similar ya que no se encontraron diferencias significativas, $(p<0,05)$.

Cuadro 3. Índice de Shannon Weaver para clusters de buena, mediana y mala calidad de agua.

\begin{tabular}{|l|l|}
\hline Clusters & Índice Shannon Weaver (media $\pm \mathrm{EE}$ ) \\
\hline Buena Calidad & $0.73 \pm 0.19 \mathrm{~b}$ \\
\hline Mediana Calidad & $0.32 \pm 0.07 \mathrm{a}$ \\
\hline Mala Calidad & $0.23 \pm 0.12 \mathrm{a}$ \\
\hline
\end{tabular}

Letras diferentes entre columnas indican diferencias significativas. Duncan $(p<0,05)$

$\mathrm{Al}$ analizar el componente de protección en las quebradas se encontró que la diversidad biológica en las quebradas protegidas y no protegidas fue diferente significativamente $(\mathrm{p}<$ 0,05 ), habiendo mayor diversidad en las protegidas (Cuadro 4). Esto confirma los resultados encontrados en este mismo estudio. En la Ilustración 4 a y b se ve claramente que según el índice BMWP, las quebradas protegidas y con pasturas mejoradas con alta densidad de árboles tienden a mejorar la calidad del agua, lo que hará que exista una mayor diversidad de especies indicadoras de buena calidad de agua.

Cuadro 4. Índice de Shannon Weaver para quebradas protegidas y no protegidas

\begin{tabular}{|l|l|}
\hline Quebradas & Índice Shannon Weaver (media $\pm \mathrm{EE}$ ) \\
\hline Protegido & $0.5 \pm 0.09 \mathrm{a}$ \\
\hline No protegido & $0.2 \pm 0.08 \mathrm{~b}$ \\
\hline
\end{tabular}

Letras diferentes entre columnas indican diferencias significativas. Duncan $(\mathrm{p}<0.05)$. 
a

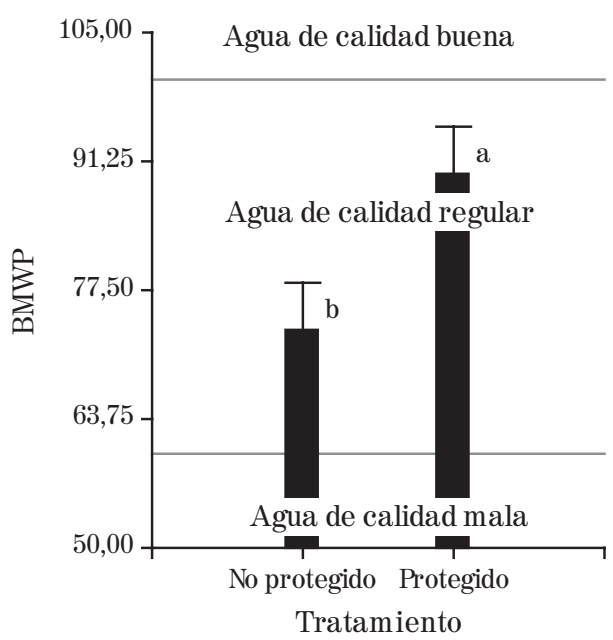

$\mathrm{b}$

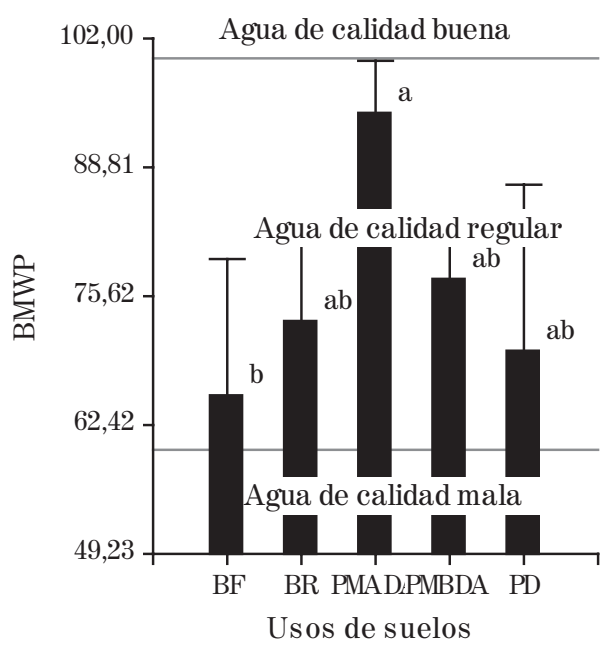

Letras diferentes entre columnas indican diferencias significativas. Duncan $(\mathrm{p}<0.05)$. $\mathrm{BF}=$ banco forrajero, $\mathrm{BR}=$ bosque ripario, $\mathrm{PMADA}=$ pastura mejorada con alta densidad de árboles, $\mathrm{PMBDA}=$ pastura mejorada con baja densidad de árboles, $\mathrm{PD}=$ pastura degrada.

Ilustración 4. a) Calidad de agua según índice BMWP para quebradas protegidas y no protegidas. b) Calidad de agua según índice BMWP en los diferentes usos de suelo evaluados en fincas ganaderas de Matiguás, Matagalpa, Nicaragua.

\section{Conclusiones}

La calidad del agua en las microcuencas ganaderas estudiadas en los ríos Paiwas y Bul Bul señala que éstas son aguas no aptas para consumo humano, aunque sí para otras actividades productivas.

Los usos de suelo que incorporan árboles a diferentes densidades y con menor intervención del hombre mejoran el hábitat albergando mayor diversidad de especies indicadoras de aguas de buena calidad.

Los puntos de muestreo que presentan mala calidad de agua y que tienen bosque ripario (BR) están intervenidos directamente por la presencia constante del ganado, por lo que la franja de bosque que protege al cuerpo de agua no evita la contaminación, ya que hay deposición directa de materia fecal en el agua.

La baja diversidad de macroinvertebrados en los bancos forrajeros (BF), lo que indica mala calidad del agua, está influenciada por la época de corte y acarreo, extracción de grandes cantidades de agua para la regeneración del material vivo y una mayor intervención del hombre, lo que conlleva a la modificación del hábitat. 
Las pasturas degradadas presentaron hábitats muy pobres para la colonización, lo que limita la diversidad de los macroinvertebrados, siendo éstas dominadas por especies indicadoras de mediana y mala calidad de agua.

\section{Referencias bibliográficas}

AUQUILLA, R. (2005). Uso del suelo y calidad del agua en quebradas de fincas con sistemas silvopastoriles en al sub cuenca del río Jabonal, Costa Rica. Tesis Mgs Sc, Centro Agronómico Tropical de Investigación y Enseñanza (CATIE): Turialba, Costa Rica, 123 p.

CHARÁ, J. (2003). Manual para la evaluación biológica de ambientes acuáticos en microcuencas ganaderas. CIPAV: Cali, Colombia.

DE LA LANZA, G.; HERNÁNDEZ-PULIDO, S. \& CARVAJAL, J.L. (2000). Organismos indicadores de la calidad del agua (BIOINDICADORES). IBUNAM y Plaza Valdés: México.

FIGUEROA, R, et al, (2003). "Macroinvertebrados bentónicos como indicadores de calidad de agua de ríos del sur de chile".En Revista chilena de historia natural. Volumen 76 n.2: Santiago.

GARCÍA, L. (2003). Indicadores técnicos y evolución de la influencia del uso de la tierra en la calidad del agua, subcuenca del Río Tascalapa, Yoro, Honduras. Tesis Mag. Sc. CATIE/UCR: Turrialba, Costa Rica.136p.

GUERRERO, A. \& SORIANO, C. (1992). Monografías de Matagalpa, en línea. 27p. Disponible en:www.inifom.gov.ni/caracter/información/Matagalpa/matiguas. (consultado en febrero de 2006).

ROLDÁN, G. (1988). Guía para el estudio de los macroinvertebrados acuáticos del departamento de Antioquia. Fondo FEN Colombia, COLCIENCIAS, Universidad de Antioquia: Antioquia, Colombia.

RUÍZ, A. (2002). Fijación y almacenamiento de carbono en sistemas silvopastoriles y competitividad económica en Matiguás, Nicaragua. Tesis Mag. Sc. Centro Agronómico Tropical de Investigación y Enseñanza CATIE: Turrialba, Costa Rica.106p.

SHANNON, C.E. \& WEAVER, W. (1949). The Mathematical Theory of Communication. University of Illinois Press: Urbana, Illinois. 